\title{
A diversity with several levels: Kenyan politics of integration and the Kenyan minorities of Indian origin
}

Michel Adam

\section{(2) OpenEdition}

Journals

Electronic version

URL: https://journals.openedition.org/eastafrica/402

DOI: $10.4000 /$ eastafrica.402

ISSN: 2790-1076

Publisher

IFRA - Institut Français de Recherche en Afrique

Printed version

Date of publication: 1 September 2013

Number of pages: $23-32$

ISSN: 2071-7245

Electronic reference

Michel Adam, "A diversity with several levels: Kenyan politics of integration and the Kenyan minorities of Indian origin", Les Cahiers d'Afrique de l'Est / The East African Review [Online], 47 | 2013, Online since 07 May 2019, connection on 09 December 2021. URL: http://journals.openedition.org/eastafrica/402 ; DOI: https://doi.org/10.4000/eastafrica.402 


\title{
L'influence des corridors dans le développement régional : le cas de l'East African Community
}

\author{
Ronan Porhel \& Alain Léon
}

\section{Introduction}

Bien que l'EAC suive fidèlement les grandes étapes de l'intégration institutionnelle (de l'union douanière à l'union politique), cette organisation aspire également à développer et à améliorer la coopération sectorielle de la région et ce à l'image la Communauté de développement de l'Afrique Australe (SADC). La volonté de se doter de structures institutionnelles indispensables à la création d'un environnement commun s'accompagne donc incontestablement par la mise en place de projets sectoriels régionaux tels que la gestion de cet espace commun que représente le lac Victoria ou bien la réhabilitation des corridors de transport : le corridor nord de Mombasa à Kampala via Malaba ; le corridor central de Dar es Salaam à Kigoma via Tabora et en Ouganda via Masaka et le corridor sud de Dar es Salaam à Lusaka via Mbeya.

Le commerce des pays de l'EAC avec le reste du monde étant nettement plus important que le commerce inter-régional, la dépendance des États enclavés ${ }^{1}$ vis-à-vis des ports de l'Océan indien, et donc des corridors, est devenue un enjeu crucial. Ces infrastructures de transports permettent par ailleurs de développer la complémentarité des activités au sein du processus d'intégration régionale. Pourtant, malgré l'existence d'importants corridors, force est de constater que ces derniers perdent de plus en plus de leur compétitivité par la hausse régulière des coûts. Le risque est que les entreprises de la région perdent des marchés et ne s'adaptent plus à la concurrence mondiale. Quelles solutions vont alors être apportées par les États membres de l'EAC et quel rôle peut jouer l'institution régionale?

Si le système de corridors est-africain a effectivement participé au développement de la région, il montre aujourd'hui de nombreuses limites (1). Dans une perspective d'avenir, afin de pallier ces difficultés, les États membres prévoient des investissements d'ampleur lesquels seraient plus efficaces avec un approfondissement de l'accord régional (2).

1 Les pays enclavés sont l'Ouganda, le Rwanda, le Burundi mais également les autres pays d'Afrique centrale comme l'Est de la République Démocratique du Congo (RDC) et désormais le Soudan du sud ou l'Éthiopie. 


\section{Les corridors d'Afrique de l'Est : un outil d'intégration régionale indispensable mais qui reste imparfait}

Conformément à l'intégration régionale des espaces, la création d'infrastructures « interconnectantes » a favorisé le dynamisme commercial de la région et l'éclosion de nouvelles activités (1.1.). Mais les perturbations chroniques le long des corridors ainsi que l'incapacité à développer certaines régions révèlent les insuffisances de cet outil (1.2.).

\subsection{La création d'un espace commun basé sur des corridors}

\section{De la nécessité de développer des corridors}

Un corridor de transport est une structure multimodale composée d'axes routiers, ferroviaires, fluviaux ou encore aériens, fournissant alors une connexion économiquement efficiente entre des centres d'activités économiques d'une ou plusieurs zones enclavées d'un ou de plusieurs pays à un pays voisin ayant un accès maritime. Les corridors les plus modernes se composent également des oléoducs, gazoducs, des lignes à haute tension ou de la fibre optique. Aussi, la région de l'EAC connait un système de corridors développé depuis la période coloniale britannique.

Dans son processus de développement, l'EAC a intégré une union douanière en 2005 puis un marché commun en 2010. Mais l'élaboration de cette communauté économique régionale (CER) s'inscrit également dans l'intégration d'un espace commun lequel produit un double effet d'agglomération et de diffusion tel qu'évoqué par Paul Krugman dans la nouvelle économie géographique ${ }^{2}$. Aussi une région défavorisée, désirant être reliée à un processus de développement en cours, doit créer les conditions de son attractivité en s'attachant à instaurer un "capital public spatialisé $»^{3}$. Compte tenu des forts coûts de transports dans les pays en développement (PED), en particulier à destination des pays enclavés ${ }^{4}$, les principales institutions internationales, dont la Banque Mondiale, ont reconnu l'impact bénéfique des investissements en infrastructures "interconnectantes » entre différents territoires sur la croissance des pays africains ${ }^{5}$.

\footnotetext{
2 La nouvelle économie géographique montre le rôle décisif de l'espace dans l'émergence d'économies d'agglomération en raison de la concentration de producteurs d'une même branche. À ce titre elle rejoignait les conclusions de G. Myrdal sur le concept de croissance déséquilibrée par une phase de concentration puis de diffusion. Voir Krugman, P., «Increasing returns and economic geography », Journal of Political Economy, nº99, Vol. 31, 1991, pp. 483-499.

3 Le capital public spatialisé peut être représenté par des services publics comme les transports, les télécommunications, la recherche etc. Voir Akanni-Honvo, A. \& Léon, A., « La croissance endogène régionalisée ", Revue Tiers Monde, $\mathrm{n}^{\circ} 155$, juillet-septembre, 1998 , Paris.

4 Selon la Banque Mondiale, les coûts de transport dans les pays enclavés peuvent atteindre $77 \%$ de la valeur des biens exportés. Avant d'être à bord d'un navire, un conteneur coûte en moyenne 872 Dollars US (USD), soit $703 €$ en transport, contre 300 USD en Chine.

5 Selon une étude de la Banque Mondiale, un taux de croissance annuel de $13 \%$ des infrastructures est corrélé à 1 point de croissance économique par tête. Voir « World Bank, The Africa Competitiveness », World Economic Forum Report, 2013.
} 
Dès la période 2001-2005, la stratégie de l'EAC, ayant pour but de faire participer l'ensemble des territoires à la dynamique régionale, a donc été de tisser un maillage d'infrastructures du local au régional. Ces interconnexions doivent permettre de créer un grand marché susceptible d'exploiter toutes les potentialités de la zone en terme de complémentarité des structures productives (Kenya : place boursière et main d'œuvre qualifiée ; Tanzanie : ressources naturelles ; Ouganda : hydroélectricité, etc.). Dans ce contexte, les États partenaires espèrent attirer les investisseurs étrangers pour financer de nouvelles activités.

\section{L'espace est-africain s'appuie sur trois corridors majeurs :}

L'espace est-africain se structure autour de deux axes principaux, le corridor nord (de Mombasa à Kampala via Malaba) et le corridor sud (Dar es Salaam à Lusaka via Mbeya), intercalé par un troisième couloir commercial le corridor central (Dar es Salaam à Kigoma via Tabora et en Ouganda via Masaka) ${ }^{6}$. Les dynamiques régionales étant depuis longtemps tournées vers l'exportation de matières premières, l'essentiel des richesses se produisent le long de ces corridors et l'entité est-africaine s'est créée à partir de cet $\mathrm{ADN}^{7}$.La réactivation de ces trois couloirs commerciaux a vite constitué une priorité pour rendre ces espaces plus communicants et réduire des coûts de transaction très élevés.

Le corridor nord, de loin le plus fréquenté, traverse l'intérieur du Kenya de Mombasa à Malaba via Nairobi. Après le poste frontière de Malaba, le corridor s'éclate en deux segments, l'un prenant la direction de Kampala (Ouganda), du Rwanda et de la République Démocratique du Congo et l'autre allant vers le Sud Soudan et l'Éthiopie. Point de transit incontournable, aussi bien pour les importations que pour les exportations ${ }^{8}$, le port de Mombasa est raccordé aux principales villes de l'hinterland, Kampala en Ouganda, Kigali au Rwanda, Bujumbura au Burundi ainsi qu'à Goma et Kisangani en République Démocratique du Congo (RDC). Entre 2007 et 2009, l'activité du port de Mombasa a augmenté de $40 \%$, ( $+30 \%$ pour les containers) en raison du dynamisme des pays enclavés et de l'apparition du Sud-Soudan comme nouveau partenaire. Les 1898 kilomètres du corridor nord sont en très grande partie bitumés sur une seule voie 9 . Par ailleurs, une concession importante du réseau ferré de Kenya Railway et Uganda Railway a été accordée à un exploitant privé sud-africain, la Rift Valley Railway (RVR) en 2005. Cette concession est cependant effective seulement depuis 2010. En raison de défauts d'entretien et de pénuries d'équipements, seul $5 \%$ du trafic de ce corridor utilise ce mode de transport. Un oléoduc jusqu'à la ville d'Eldoret et un système de voies navigables sur le lac Victoria viennent compléter la structure. Un

\footnotetext{
6 Voir carte de l'EAC et ses corridors en annexe 2.

7 Selon Hervé Maupeu, politiste et spécialiste de l'Afrique orientale à l'Université de Pau et des Pays de l'Adour. $895 \%$ des marchandises qui sont importées vers - ou exportées de - l'Ouganda transitent par le port de Mombasa. Voir Trade Facilitation in the East African Community: Recent Developments and potential Benefits, US International Trade Commission. Publication n ${ }^{\circ} 4335$. July 2012.

9 Selon une étude menée, en 2010, par la firme Aurecon Engineering pour le East African Transport Strategy and Regional Road Sector Development Program, les trois quarts du réseau routier du corridor nord sont notés «acceptables » à « bons »; mise à part le segment reliant le Rwanda et le Burundi.
} 
début d'allègement des procédures aux trois postes frontières ${ }^{10}$, ainsi que la modernisation des installations, ont permis de diviser par deux environ les distances économiques entre 2005 et $2011^{11}$. Pour certains chefs d'entreprise comme Robert Byaruhanga, responsable de la logistique au sein de l'entreprise ougandaise Kyagalanyi Coffee Ltd., les améliorations du corridor nord ont d'ores et déjà eu des retombées bénéfiques et permettent de réaliser des économies sur les frais de transit : "Cela prenait tellement de temps avant, il fallait en moyenne compter 40 à 50 jours pour faire expédier un chargement de café de Kampala jusqu'à un bateau. Mais, aujourd'hui, nous nous en sortons en 18-23 jours ».

Le corridor sud, composé d'un réseau routier, ferroviaire sur l'ensemble de la distance et d'un oléoduc jusqu'à Ndola, raccorde le port de Dar es Salaam en Tanzanie à Lusaka en Zambie via Mbeya. L'écartement austral (1,6 mètres) de la voie ferrée de la compagnie Tazara permet de relier les pays membres de la Communauté de développement d'Afrique Australe (SADC) au port tanzanien.

Le corridor central représente le principal hub d'échanges pour la Tanzanie et les deux derniers adhérents de l'EAC, le Burundi et le Rwanda ${ }^{12}$ mais reste, pour le moment, marginal pour l'Ouganda en raison d'un coût peu compétitif. Il relie Dar es Salaam à Kigoma via Tabora et fait la jonction avec l'Ouganda via Masaka par la route ou par voie maritime à travers le lac Victoria entre Mwanza et Port Bell. La voie ferroviaire s'arrêtant à Mwanza, la liaison se fait essentiellement par un réseau routier désormais bitumé à $86 \%{ }^{13}$. Par contre, comme son homologue du nord, le corridor est constitué d'une seule voie sur $90 \%$ de leurs parcours respectifs ${ }^{14}$. Entre 2006 et 2009, l'activité du port de Dar es Salaam, pourtant moitié moins importante que celui de Mombasa, s'est accrue de $48 \%$ ( $+22 \%$ pour les containers) ${ }^{15}$.

10 Les trois postes frontières du corridor nord se situent à Malaba (Kenya-Uganda), Gatuna-Katuna (OugandaRwanda), and Akyaru-Kinyaru Haut (Rwanda-Burundi). Entre 2005 et 2011, mise à part le cas du Burundi les documents à fournir pour les importateurs et les exportateurs ont nettement baissé : 4 pour le Kenya, 18 pour le Rwanda, 9 pour la Tanzanie et 15 pour l'Ouganda. En 2011, le nombre de documents exigé pour les importateurs, par exemple, s'élève à 6 pour la Tanzanie et 10 pour le Burundi. Voir Trade Facilitation in the East African Community: Recent Developments and Potential Benefits, op. cit.

11 Selon le «Trade Mark East Africa » (TMEA), institution responsable de l'observation des principaux corridors de la région sous l'égide du programme des politiques de transport en Afrique Sub-saharienne (SSATP), entre 2005 et 2011, en moyenne, les délais de passage de la frontière à Malaba ont été réduits de plus de 3 jours à 3-5 heures. Le temps d'immobilisation au port de Mombasa est réduit de 19 à 8 jours et le temps de transit le long du tronçon Mombasa-Nairobi-Kampala a baissé de 15 à 5 jours.

$1257 \%$ des importations et $65 \%$ des exportions du Rwanda passent par le corridor central. Les chiffres sont respectivement $94 \%$ et $98 \%$ pour le Burundi. Voir Trade Facilitation in the East African Community..., op. cit.

13 Étude menée, en 2010, par la firme Aurecon Engineering pour le East African Transport Strategy and Regional Road Sector Development Program.

14 Trade Facilitation in the East African Community ..., op. cit.

15 Entre 2007 et 2009, les volumes traités au port de Mombasa sont passés de 10,7 à 15 millions de tonnes dont 30\% de containers. Entre 2006 et 2009 les volumes échangés au port de Dar es Salaam sont passés de 7,2 à 10,6 millions de tonnes. Voir Society for International development (SID), The State of East Africa 2012. Deepening Integration, Intensifying Challenges. Nairobi, SID, 2012. 
Ce système structuré entre le hub aéroportuaire de Nairobi, les deux grands ports de l'Océan indien (Mombasa et Dar es Salaam) et des villes intermédiaires servant de lieux de liaison, a amené les acteurs à considérer cette zone comme une seule et même unité tarifaire anticipant dès lors la vision régionale de $1^{\prime} \mathrm{EAC}{ }^{16}$. Si des voies de communication sommaires restent encore assez fournies entre ces trois principaux segments, les versants nord et sud de l'Afrique Orientale se particularisent cependant par l'extrême pauvreté de l'infrastructure. En concentrant ses dépenses sur les zones à fort potentiel, situées autour de Nairobi, les autorités kenyanes ont délaissé les axes nord $/ \mathrm{sud}^{17} \mathrm{mise}$ à part la portion de route Arusha-Nairobi via Namanga. Le quadrillage de la région est donc loin d'être atteint. Seul $9 \%$ du réseau de l'EAC est bitumé.

\section{Un commerce en hausse grâce à l'apport des corridors}

La réhabilitation de liaisons routières infra-nationales et régionales d'une part, et la mise en œuvre de l'union douanière d'autre part, se sont traduites par des effets d'agglomération autour des points de connexion et des créations de commerce. Le commerce entre les pays de 1'EAC a presque doublé entre 2005 et 2010 en passant de 2,2 à 4,1 milliards USD. Le commerce régional avec le reste du monde a augmenté encore plus vite, signe de la vitalité des corridors de transport.

\section{Les exportations de l'EAC et le commerce intra-régional}

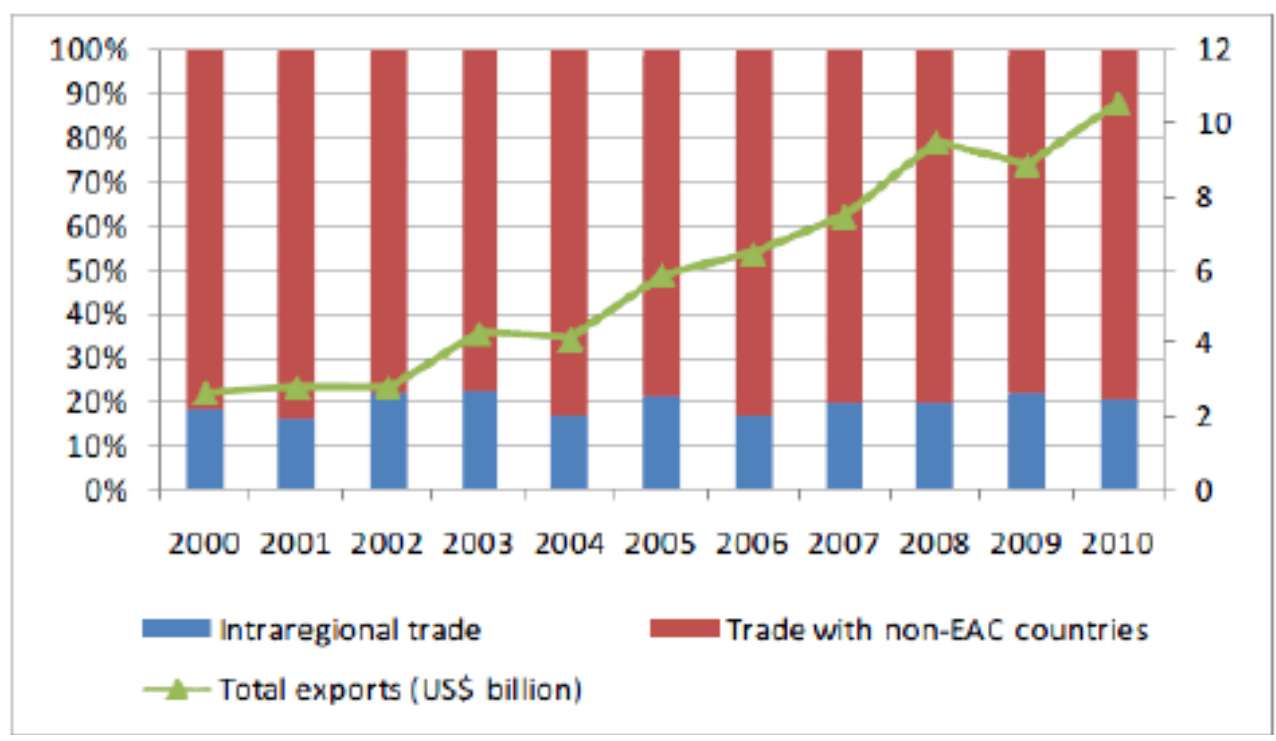

Source: United Nations Comtrade via World Integrated Trade Solutions

16 Calas, B. « Le système logistico-urbain est africain », Mambo!, IFRA, 2000.

17 En l'absence de connexions de qualité, les surplus alimentaires du sud et de l'ouest de la Tanzanie peuvent difficilement combler les déficits des régions nord du Kenya, de l'Ouganda mais également de la Somalie et de l’Éthiopie. 
Ce développement du commerce s'est accompagné d'une hausse des débouchés et d'une diversification des exportations grâce au dynamisme de nouvelles activités. Même si les productions périssables à forte valeur ajoutée sont plutôt utilisatrices du transport aérien, celles-ci ont également besoin de s'appuyer sur un réseau routier performant. C'est le cas des secteurs de la pêche et surtout de l'horticulture. À la différence des pêcheries tanzaniennes et ougandaises qui empruntent les aéroports de proximité de Mwanza et d'Entebbe, celles localisées autour de Kisumu doivent exporter leurs filets de perche, à partir de l'aéroport de Nairobi, à plus de 300 kilomètres. La modernisation et le prolongement du corridor nord ont donc permis de respecter davantage les délais à l'export et d'élargir le marché local et régional, pour les « bas morceaux », jusqu'au cœur de l'Afrique centrale et au Sud Soudan.

L'horticulture est un secteur prometteur pour ses perspectives de croissance et les dotations naturelles dont sont pourvues les pays de la zone ${ }^{18}$. L'essentiel des cultures horticoles, incluant les fruits, les légumes et les fleurs coupées, sont concentrées dans la région du lac Naivasha au Kenya, autour d'Arusha et de Moshi en Tanzanie. Mais du fait de leur extrême fragilité, elles doivent avoir atteint leur point de vente, local ou international, dans les 24 heures suivant la cueillette. La modernisation du réseau routier du corridor nord et de la liaison Arusha Nairobi via Namanga étaient des conditions pour que ces produits à forte valeur ajoutée puissent être exportés au plus vite à partir de l'aéroport de Nairobi. Selon le « Kenya Flower Council », le secteur a rapporté en 2011, 1,087 milliards USD devenant le plus important générateur de devises. Lors des quatre années précédant la crise économique mondiale, le secteur horticole en Tanzanie a connu une croissance de $8 \%$ à $10 \%$ par an.

\subsection{Un système de corridors toujours faillible}

Si le corridor nord a permis la concentration d'activités dans un rayon de 100 kilomètres autour de la ligne ferroviaire reliant le Kenya à l'Ouganda ${ }^{19}$, il a également accentué la marginalisation économique des communautés vivant dans les régions arides du nord et les zones rurales et a échoué à rendre attractives les zones portuaires de 1'Océan indien. Ainsi, malgré le constat d'une hausse du revenu par habitant dans la région, le nombre de personnes vivant sous le seuil de pauvreté, moins de 1,25 USD par jour selon la Banque mondiale, a augmenté de 44 à 53 millions de personnes entre 1994 et 2010 pour atteindre $38 \%$ de la population de la région ${ }^{20}$. Les trois comtés de l'extrême nord du pays sont, de loin, les plus pauvres du pays. 94,3\% des habitants du Turkana vivent sous le seuil de pauvreté, contre 22\% dans la capitale Nairobi, selon le dernier recensement de 2009.

18 Situé aux abords de l'équateur, le secteur horticole de ces pays peut fonctionner toute l'année.

19 Déclaration de M. Cyrus Njiru, Secrétaire Permanent de l’EAC aux Transports en juin 2012. www.

theafricareport.com

20 Society for International Development (SID), The State of East Africa 2012..., op.cit. 
Par ailleurs, malgré les améliorations enregistrées depuis les années 2000, les États membres ont obtenu de faibles indices de performances logistiques ${ }^{21}$ dus notamment au retard de compétitivité des transports régionaux ${ }^{22}$. Comparé aux USA et à l'Europe, le surcoût par kilomètre s'élève encore à $50 \%$ pour les deux corridors et même à $75 \%$ pour les pays enclavés ${ }^{23}$. Une étude du Conseil kenyan des transporteurs (KSC) a révélé qu'un conteneur de 40 pieds nécessite 28 jours de Shanghai (Chine) à Mombasa et coûte 6000 USD, alors que le même conteneur nécessite 41 jours de Mombasa à Bujumbura pour 8000 USD.

Indices de performance logistique dans l'EAC (cf. paragraphe précédent)

\begin{tabular}{|c|c|c|c|c|c|c|c|}
\hline & \multicolumn{5}{|c|}{ EAC } & \multirow{2}{*}{$\begin{array}{r}\begin{array}{r}\text { Top low- } \\
\text { income } \\
\text { countryb }\end{array} \\
\text { Benin } \\
\end{array}$} & \multirow{2}{*}{$\begin{array}{r}\text { Bes } \\
\text { practices } \\
\text { country } \\
\text { Singapo'e }\end{array}$} \\
\hline & Burundi & Kenya & Rwanda & Tanzania & Uganda ${ }^{a}$ & & \\
\hline \multicolumn{8}{|l|}{$\begin{array}{l}\text { Logistics performance index scores } \\
\text { on a scale from } 1 \text { (wcrst) to } 5 \text { (best) }\end{array}$} \\
\hline Aggregate score & 1.61 & 2.43 & 2.27 & 2.65 & $2.82^{*}$ & 285 & 4.13 \\
\hline Efficiency of customs procedures & 1.67 & 2.08 & 2.19 & 2.17 & $2.84^{*}$ & 259 & 4.10 \\
\hline Percentage of cargo inspected & $60 \%$ & $25 \%$ & (9) & (9) & $75 \%$ & $11 \%$ & $1 \%$ \\
\hline Infrastructure quality & 1.68 & 2.16 & 1.88 & 2.41 & $2.35^{*}$ & 257 & 4.15 \\
\hline Ease of shipping internationally & 1.57 & 2.69 & 2.27 & 2.91 & $3.02^{*}$ & 2.44 & 3.99 \\
\hline Timeliness of deliveries & 1.67 & 2.88 & 2.76 & 2.97 & $3.52^{*}$ & 3.74 & 4.39 \\
\hline \multicolumn{8}{|l|}{ Trading across borders indicators } \\
\hline Documents needed to import & 10 & 7 & 8 & 6 & 9 & 8 & 4 \\
\hline Documents needed to export & 9 & 3 & 8 & 6 & 7 & 7 & 4 \\
\hline Days needed to import & 54 & 24 & 31 & 24 & 34 & 32 & 4 \\
\hline Days needed to export & 35 & 26 & 29 & 18 & 37 & 30 & 5 \\
\hline
\end{tabular}

Source. World Bank, "Logistics Performance Index," 2010, 2012; World Bark, "Doing Business," 2012.

"Starred "Logistics Performance" data for Uganda are from the 2010 report. All other data are from the 2012 version.

bBenin was the highest-ranked low-ncome country in the 2012 Logistics Performance Index. It was ranked ninth among low income ccuntries for Doing Business' Trading Across Borders rankings in 2012.

'Not available

21 Sur une échelle de 1 à 5, les indices de performance logistique des pays de l'EAC sont de 1,61 pour le Burundi à 2,82 pour l'Ouganda, en deçà de la moyenne des pays de l'Afrique sub-saharienne. Construit par la Banque Mondiale, l'indice de performance de la logistique reflète les perceptions relatives à la logistique d'un pays basées sur l'efficacité des processus de dédouanement, la qualité des infrastructures commerciales et des infrastructures de transports connexes, la facilité de l'organisation des expéditions à des prix concurrentiels, la qualité des services d'infrastructure, la capacité de suivi et de traçabilité des consignations et la fréquence avec laquelle les expéditions arrivent au destinataire dans les délais prévus.

22 Les coûts de transport depuis le port de Mombasa : 0,04 USD/km pour le Kenya ; 0,085 USD pour l'Ouganda ; 0,09 USD pour le Rwanda et 0,11 USD pour Burundi. Voir Transport Prices and Costs in Africa; BIRD, World Bank, 2009.

23 Trade Facilitation in the East African Community..., op. cit. 
Ce problème de compétitivité des transports est lié notamment aux dysfonctionnements des deux portes océanes de l'EAC (Mombasa et Dar es Salaam) ainsi qu'à la présence de barrières non tarifaires, le long des corridors. Ces éléments sont en effet en grande partie responsables du ralentissement du trafic et de l'incertitude relative au commerce en général.

Concernant le dysfonctionnement portuaire (des deux portes océanes), le port de Mombasa, conçu à l'origine pour accueillir 250000 conteneurs (EVP), en a traité, en 2012, plus de $800000^{24}$. Cet engorgement contraint les bateaux à stationner avant d'accoster, près de 15 jours, à un coût journalier entre 10000 et 12000 USD, Mais pour Wolfgang Fengler, chef économiste de la Banque Mondiale au Kenya, le principal problème provient surtout de l'inefficacité des installations portuaires ${ }^{25}$. À Mombasa, une multitude d'organisations ${ }^{26}$ présentes sur le port et dont on ne connait pas toujours les véritables responsabilités, ralentissent non seulement le traitement des containers mais favorisent également les tentations de corruption ${ }^{27}$. Le site se trouve par conséquent rapidement saturé d'autant plus que certains importateurs utilisent le port comme lieu de stockage. Cette situation toucherait d'ailleurs davantage les produits des pays enclavés. $75 \%$ des importations ougandaises sont inspectées, contre $25 \%$ pour celles à destination du Kenya, alors que les produits ont, pour la plupart d'entre eux, déjà été contrôlés par des laboratoires accrédités ${ }^{28}$. Outre cette impression de discrimination ${ }^{29}$, ces pays sans littoral subissent déjà une combinaison interactive de facteurs très variés ${ }^{30}$ qui contraignent le positionnement de leurs productions sur les marchés mondiaux et surtaxent les produits importés. La Banque Mondiale estime que le temps de dédouanement dans ce port est sept fois moins prévisible que dans les autres régions. Cette inefficacité nécessite $50 \%$ à $80 \%$ de temps en plus pour les importateurs des pays enclavés.

A la sortie du port, les corridors routiers sont largement plus utilisés ${ }^{31}$ que le réseau ferroviaire pourtant plus économique à la tonne-kilomètre $(0,05$ USD par kilomètre pour une tonne contre 0,09 USD par la route). Mais la mauvaise qualité du réseau le rend assurément plus imprévisible.

24 «Congestion at Mombasa port slows down trade in EAC bloc », The East African, 13 décember 2012.

25 Selon Jacques Morisset, chef économiste de la Banque Mondiale en Tanzanie, l'inefficacité du port de Dar es Salaam pourrait être à l'origine d'une perte de 1,8 milliards USD pour le pays soit $7 \%$ du PIB. Voir «Inefficiency at Dar port swallows up \$2,6 bn of GDP», The East African, 25 mai 2013.

26 The Kenya Ports Authority (KPA), Kenya Revenue Authority (KRA), Kenya Bureau of Standards, Kenya Plant Health Inspectorate Services, Port Health Authority (PHA), Dairy Board of Kenya, National Bio-Safety Authority, Anti-Counterfeit Agency and Port Police.

27 Plusieurs scandales durant le régime Kibaki ont montré que ces filières de corruption à partir du port de Mombasa sont toujours au cœur du système clientéliste des élites kenyanes.

28 Trade Facilitation in the East African Community: Recent Developments and potential Benefits, op. cit.

29 World Bank, East African Community. Reshaping Economic Geography of East Africa: From Regional to Global Integration, Report nº5699-AFR, June 2012.

30 Ces facteurs sont : la géographie physique (distances ; climat ; densité végétale, systèmes orographiques) ; les performances logistiques (état des infrastructures, traçabilité) ; les problèmes politiques et humains (gouvernance, corruption, barrages illégaux, instabilité politique et ethnique) et enfin le cadre réglementaire. Voir Alix, Y. \& Pelletier, J.-F., «Territoires enclavées et opportunités de marché : analyse des performances logistiques des corridors de transport en Afrique subsaharienne », Organisations et territoires, 2011.

31 Selon Polycarp Igathe, chairman of the Kenya Association of Manufacturers : "le port de Mombasa est peut-être le seul où $95 \%$ des bateaux sont évacués par la route. C'est totalement insoutenable". 
Cependant, sur les routes, un problème de taille se pose depuis plusieurs années : les nombreuses barrières non tarifaires. Elles allongent considérablement la durée du trajet entrainant des coûts annexes ainsi qu'une baisse de compétitivité des entreprises. Ces barrières peuvent revêtir différentes formes, tel que le manque d'harmonisation des procédures aux différents postes frontières, tel aussi la mise en place de " ponts à bascules " pour préserver l'infrastructure routière en stoppant à chaque arrêt les camions pendant près de trois heures ${ }^{32}$, ou encore le nombre notable de barrages routiers souvent informels ${ }^{33}$. Malgré la volonté ces dernières années de réduire ces barrières non tarifaires, notamment par l'instauration du système informatique de partage de fichiers douaniers Radex et d'opérations de gestion aux postes frontières (OSBP), le corridor nord continue pourtant de les développer. En 2013, 26 jours sont en moyenne nécessaires pour importer un container en Ouganda contre 24 jours l'année précédente. Par cette dégradation du commerce transfrontalier, le World Bank's Ease of Doing Business survey a abaissé le rang

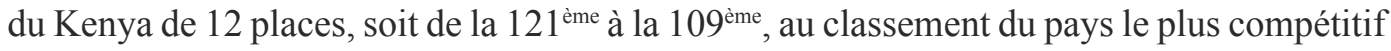
au monde ${ }^{34}$.

Particulièrement sensible à l'imprévisibilité de la durée des transports, le secteur du textile, confronté à la congestion des deux ports de l'Océan indien est contraint d'anticiper et donc d'accroître ses stocks de tissus et de divers inputs pour ne pas se retrouver en rupture de $\operatorname{stock}^{35}$. Les coûts additionnels et l'impossibilité de respecter régulièrement les délais de livraison empêchent ce secteur d'attirer les grandes firmes mondiales de l'habillement.

Cette incertitude et ces surcoûts, portés à leur paroxysme lors des violences postélectorales de 2007/8 $8^{36}$, ont d'ailleurs incité certains professionnels de l'hinterland à se tourner vers le corridor central. Le port de Mombasa a ainsi perdu, en 2011, 18,9 \% de cargaisons provenant de la Tanzanie et des autres partenaires de l'EAC au profit de celui de Dar es Salaam ${ }^{37}$. Mais la capitale économique de la Tanzanie pourrait également être menacée face à l'offensive des ports d'Afrique Australe. Sans une solution appropriée, des pays comme la Zambie, le Malawi ou la région du Katanga (est-RDC) pourraient se diriger vers le Port de Beira (Mozambique) où les prix sont plus abordables et les services efficaces.

Parallèlement à ces limites, les corridors sont également inadaptés aux nouveaux enjeux de la région. En effet la structure logistique actuelle des corridors peine à supporter une croissance du trafic de $23 \%$ entre 2007 et 2011. À ceci s'ajoute l'apparition de nouveaux partenaires, dont le Sud-Soudan, ainsi que le dynamisme des pays enclavés, notamment

32 Les pertes journalières, en raison de la contrainte des ponts à bascule, s'élèveraient à 1200 dollars par camion. Voir "The real cost of trouble at the weighbridge ", The East African Paper, 2013. 33 The 2011 Business Climate Index Survey estime à 24 le nombre de barrages routiers au Kenya et 21 en Ouganda.

34 "Kenya grants Uganda licence for Mombasa freight station ", The East African, 20 mai 2013. 35 Selon une étude de la Banque Mondiale, avec davantage de prévisibilité, les importateurs pourraient mieux gérer leurs stocks en les abaissant de 45 à $50 \%$ d'où une économie de coûts.

36 Porhel, R. «Les conséquences économiques de la crise politique », Les élections générales de 2007 an Kenya, Nairoib/ Paris, IFRA/ Karthala, 2008.

37 Africa Research Bulletin, janvier 2012. 
suite à la découverte de gisements pétroliers en Ouganda, dans les provinces orientales de la $\mathrm{RDC}$ et plus récemment autour du lac Turkana, lesquels vont contribuer dans les prochaines années à un développement des échanges de l'ordre de $8 \%$ par an. L'acheminement de ces produits pétroliers ainsi que l'électricité des barrages et éventuellement le gaz du sud-ouest de la Tanzanie vient relancer le débat sur les modes de transport.

En tout état de cause, les corridors d'Afrique de l'Est soulèvent de nombreux problèmes auxquels les États membres doivent répondre dans les meilleurs délais afin d'améliorer la compétitivité globale de la zone.

\section{Quelles perspectives?}

Face à cette situation, les autorités kenyanes et tanzaniennes ont décidé de mettre l'accent sur une modernisation de la structure logistique. L'amélioration des infrastructures régionales constitue un préalable indispensable à la diffusion de la croissance à l'ensemble des États membres et à l'interconnexion des villes motrices avec les zones marginales. Ainsi les investissements sur le territoire kenyan se sont portés sur la consolidation du corridor nord doublé par la construction d'une nouvelle voie multimodale partant de Lamu (2.1) mais les craintes des pays enclavés, vis-à-vis du pouvoir kenyan, les ont conduits à vouloir accélérer la modernisation des axes tanzaniens (2.2). Toutefois, si ces projets peuvent ajuster la structure logistique aux ambitions de la région, l'EAC ne pourra faire l'économie d'un approfondissement de sa stratégie d'intégration (2.3).

\subsection{Les projets kenyans confortent sa place de leader logistique}

L'objectif est double. D'une part, permettre au Kenya d'affirmer sa position

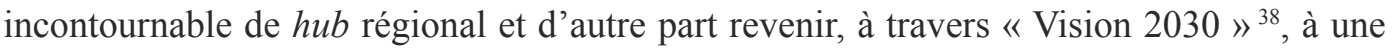
intégration par le haut. C'est-à-dire permettre aux instances de créer, à l'aide d'infrastructures, de la croissance économique et ce dans un contexte d'intense néo-libéralisme.

Dans la lignée de la rencontre tripartite entre les présidents ougandais, kenyan et rwandais à Entebbe en juin 2013, la modernisation du corridor nord doit se penser au regard de la filière pétrole que l'Ouganda souhaite développer. Ainsi, le vieux projet d'extension de l'oléoduc d'Eldoret vers Kigali via Kampala a été de nouveau étudié et ce plus de cinq ans après avoir été attribué à la société libyenne Tamoil, avec l'espoir enfin qu'il se réalise ${ }^{39}$. La construction d'une raffinerie à l'ouest de l'Ouganda est également en projet en raison des faibles capacités de celle de Mombasa. Le désengorgement du corridor nord implique un agrandissement du port de Mombasa à travers la construction d'un second terminal et par

38 «Vision $2030 »$ est un processus de développement étalé sur la période 2008-2030, à l'initiative de l'ancien président kenyan M. Kibaki, devant faire passer le Kenya au statut de pays à revenu intermédiaire. Il s'appuie sur trois piliers économique, social et politique. Il s'agit de transformer l'économie du continent en s'appuyant sur le commerce maritime et le désenclavement des pays de l'Est.

39 «Bilateral and Tripartite Deals: presidents agree to fast track political federation », The East African, 29 juin/5 juillet 2013. 